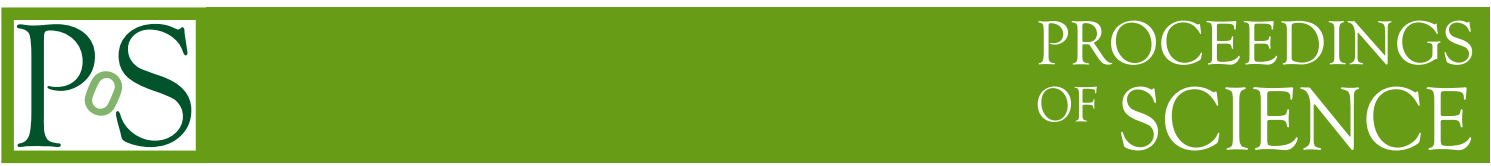

\title{
The bridge between ER Ursae Majoris-type dwarf nova and nova-like system
}

Ryoko Ishioka ${ }^{a}$, Taichi Kato ${ }^{b}$ and VSNET collaboration team

${ }^{a}$ Institute of Astronomy and Astrophysics, Academia Sinica, Taiwan

${ }^{b}$ University of Kyoto, Japan

E-mail: tkato@kusastro.kyoto-u.ac.jp, ishioka@asiaa.sinica.edu.tw

We report on the photometric observation of RZ LMi, which is a dwarf nova renowned for its extremely short ( 19 d) and regular supercycle, in 2013 and 2016. The supercycles observed in both years were longer than previously reported values of $19 \mathrm{~d}$. Especially in 2016, we observed two exceptionally long superoutbursts with drations of $28 \mathrm{~d}$ and $48 \mathrm{~d}$, which require very high mass transfer rate, $97 \%$ and $99 \%$ of the critical mass transfer rate, respectively. We consider that the object virtually experienced a transition to the nova-like state. We detected growing (stage A) superhumps with a mean period of 0.0602(1) d in 2013 and 2016, and possible negative superhumps with a period of 0.05710(1) d. By using the two periods, we estimated an orbital period of $0.05792 \mathrm{~d}$. The orbital period suggests a mass ratio of 0.10 , which is larger than mass ratios in WZ Sge-type dwarf novae with orbital periods similar to RZ LMi, and even above that of some ordinary SU UMa-type dwarf novae with similar orbital periods. The exceptionally high mass-transfer rate in this object may possibly be ecplained by a stripped secondary with an evolved core in a system evolving toward an AM CVn-type object.

The Golden Age of Cataclysmic Variables and Related Objects IV

11-16 September, 2017

Palermo, Italy 


\section{Introduction}

Dwarf novae (DNe) are a class of cataclysmic variabls (CVs) characterized by repetitive outbursts caused by a thermal-viscous instability of the accretion disk ([1]). Among DNe, there are SU UMa stars, which are characterized by long outbursts, called superoutbursts, lasting for a few weeks in addition to short normal outbursts. Short periodic modulations, so called superhumps, are observed during superoutbursts (for details of CVs, DNe, and SU UMa-type DNe. see e.g. [2]). The periods of superhumps $\left(P_{S H}\right)$ are a few percent longer than the corresponding orbital period $\left(P_{o r b}\right)$.

RZ LMi is one of the most enigmatic SU UMa-type DNe with an ultrashort (19 d) supercycle and unusually regular outburst pattern. This object was originally discovered as an ultravioletexcess variable star [3], and was confirmed as a member of the SU UMa-type DNe by the detection of superhumps ([4]; [5]) after the discovery of another SU UMa-type dwarf nova ER UMa with a short supercycle of $43 \mathrm{~d}$ ([6]) [for more details of a history of this object, see [7]]. Currently, six SU UMa-type stars including RZ LMi and ER UMa are usually called ER UMa-type stars (cf. [8]).

Though some explanations were proposed, the mechanism of the extremely short supercycle and regular outburst pattern remained mystery. [9] demonstrated that an extremely short supercycle of RZ LMi can be reproduced if the strength of tidal torques during a superoutburst is much weaker in RZ LMi than in ER UMa due to an extremely small mass ratio. [10] suggested that the tidal torque is too small to maintain the superoutburst in systems with very small $q$, and that there occurs a decoupling between tidal and thermal instabilities. These two interpretations predict the very small $q$ and the large disk radius after the superoutburst in RZ LMi. Existence of a third body is also suggested as the origin of the very regular outburst pattern ([11]). To determine the mass ratio, we need to detect the orbital period, however, RZ LMi has defied every attempt, and without $P_{\text {orb }}$ and $q$, the evolutionary status of RZ LMi remained unclear.

In this paper, we briefly reported our photometric campaigns which caught that the system became critically close to the stability border, a permanent superhumper, in 2016, and detected stage-A superhumps during two superoutbursts in 2013 and 2016 as well as possible negative superhumps and post superoutburst superhumps. Using the periods of stage-A superhumps representing the growing phase of superhumps at the radius of the $3: 1$ resonance ([12]; [13]), we obtained the relation between $R_{\text {post }}$ and $q$. We also estimated $P_{\text {orb }}$ from periods of positive and negative superhumps, and calculated the mass ratio using $P_{\text {orb }}$ and a period of stage A superhumps. Note that this paper is a summary of [7] in which more details of the results of our campaigns are published.

\section{Observations and Analysis}

Our time-resolved photometric campaigns were carried out during the periods of 2013 March 5 - April 29 and 2016 February 25 - June 10 with 33 telescopes on 29 sites. We also used the data from the AAVSO International Database, some snapshot observations between 2014 March 8 and May 22, and historical photographic data reported by [14]. The times of all observations were corrected to barycentric Julian Days (BJD). The data were analyzed in the same way as described in [15] and [16]. 


\section{Results and Discussion}

\subsection{Outburst pattern in 2013 and 2016}

Figure 1 shows the general light curves of the 2013 (upper panel) and 2016 (lower panel) observations, including six supercycles and three complete supercycles, respectively. The 4th supercycle (SC4) in 2013 was $26 \mathrm{~d}$, which was longer than the typical supercycle (20 d) of this object in 2013. The duration of the superoutburst for this supercycle was $17 \mathrm{~d}$. The $2 \mathrm{nd}$ (SC2) and 3rd (SC3) supercycles in 2016 were $32 \mathrm{~d}$ and $60 \mathrm{~d}$, which were 2-3 times longer than the reported stable supercycle $(19 \mathrm{~d})$ of this object. The durations of superoutbursts during the two supercycles were $26 \mathrm{~d}$ and $48 \mathrm{~d}$, respectively.

The existence of a stable clock such as a third body as the mechanism of the regular superoutbursts ([17];[11]) is excluded by the variable supercycles.
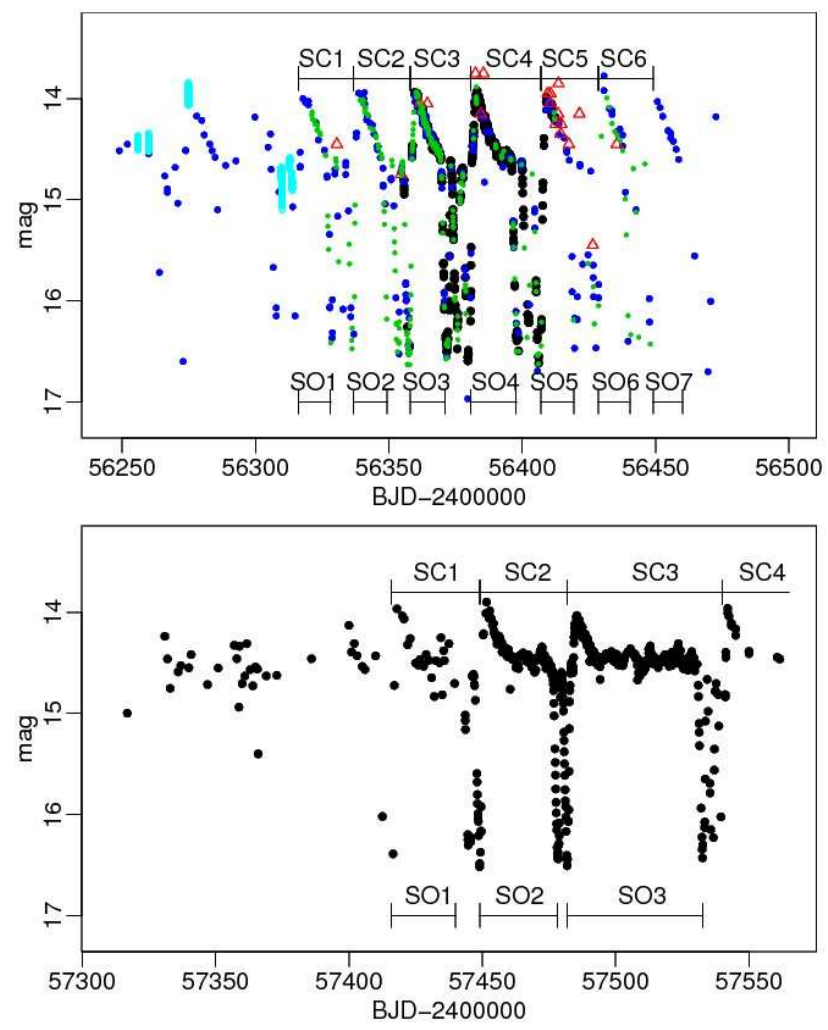

Figure 1: Overall light curves of RZ LMi of 2013 (upper) and 2016 (lower) campaigns. (E-figure1 in [7])

\subsection{Supercycle, Superoutbust Duration and Mass Transfer Rate}

One prediction of TTI model is that high mass transfer rate produces frequent outbursts with a short supercycle [18]. However, it also predicts that when the mass transfer rate becomes extremely high, supercycle lengthen again, then the system eventually reaches the "permanent outburst" state when the mass transfer rate reaches the critical value (see figure 2 in [18]). The extremely long supercycles in 2016 reproduced this prediction exactly, providing strong support to the explanation of the unusual short supercycles in ER UMa-type objects. 
The duration of a superoutburst $\left(t_{\text {supermax }}\right)$ is formulated as below [9]:

$$
\begin{gathered}
t_{\text {supermax }} \sim t_{\text {vis }}\left[f_{M} /(1-1 / e)\right]\left[1-\left(\dot{M} / \dot{M}_{\text {crit }}\right)\right]^{-1 / 2}, \\
f_{M} \sim 1-\left(R_{0} / R_{d, c r i t}\right)^{3.0}
\end{gathered}
$$

where $t_{v i s}$ is the viscous depletion timescale and $M_{c r i t}$ is the critical $\dot{M}$ required to produce a hot, stable disk, respectively. The factor $f_{M}$ is the fraction of the disk mass accreted during a superoutburst expressed by $R_{0}$ and $R_{d, c r i t}$ representing the disk radius at the end of a superoutburst and at the start of a superoutburst (assuming that the disk critically reaches the radius of the $3: 1$ resonance at the start of a superoutburst), respectively. If we assume $t_{v i s}$ and $R_{0}$ are the same between different superoutbursts, we can estimate $\dot{M}$ during each superoutburst. We take the parameters in [9], $t_{v i s}=11.2 \mathrm{~d}$ and an assumption of a large disk radius at the end of a superoutburst $R_{0}=0.42 a$, where a is the binary separation. Then, the historical shortest $t_{\text {supermax }}$ of $6 \mathrm{~d}$ [4] is reproduced with $\dot{M} / \dot{M}_{\text {crit }}=0.5$. The duration of each superoutburst in 2016 requires $97 \%$ and $99 \%$ of the critical mass transfer rate. RZ LMi was critically close to the stability border, and almost became a permanent superhumper. This object showed an almost complete transition from an ER UMa-type object to a permanent superhuper (Nova-like object). BK Lyn is another object which experienced the transitions between ER UMa-type object and a permanent superhumper ([19]; [20]).

\subsection{Growing (stage-A) superhumps}

We detected growing (stage A) superhumps at the very early phase of each superoutburst in 2013 (SC4) and 2016 (SC3). Those light curves during the stage A superhump phase are presented in Figures 2 and 3. Using the data between BJD 2456381.41 and 2456381.88, we obtained a period of 0.0602 (3) $d$ with the phase dispersion minimization (PDM:[21]) method for the 2013 April superoutburst. For the 2016 superoutburst, we obtained a period of 0.0601(1) d with the same method by using the data between BJD 2457483.01 and 2457484.40. Since these values are consistent with each other, we adopted an averaged value of $0.0602(1) \mathrm{d}$ as the period of stage A superhumps.

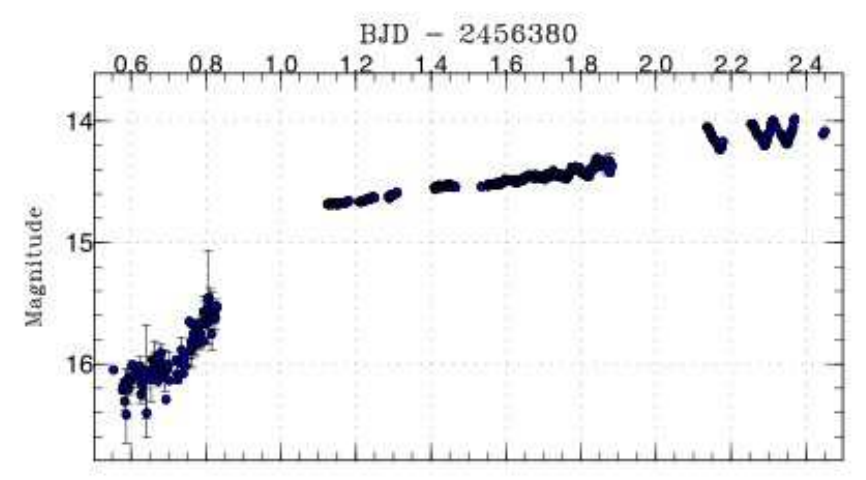

Figure 2: Growing superhumps at the start of the 2013 April superoutburst (2013-SC4).(Fig.6 in [7]) 


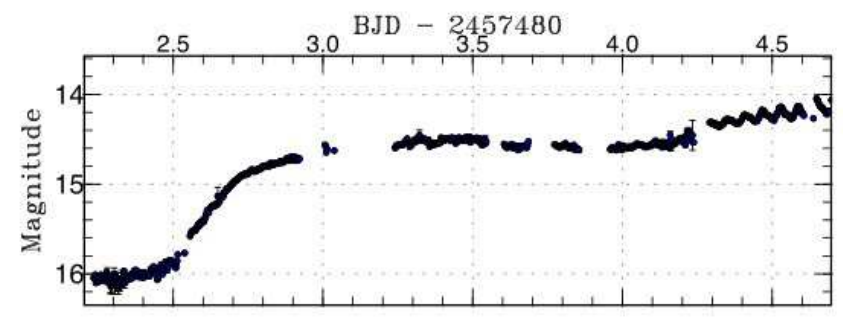

Figure 3: Growing superhumps at the start of the 2016 April superoutburst (2016-SC3). (Fig.3 in [7])

\subsection{Post-superoutburst superhumps}

On March 20, 2013, We observed the object in quiescence closely after the 2013 March superoutburst, during which the object displayed post-superoutburst superhumps with a run covering continuous six cycles. A PDM analysis of this continuous run yielded a period of 0.0594(2) d. This variation was also present on March 24, 2013, in quiescence after one further normal outburst. We combined the quiescent data on March 20 and 24 and obtained a period of 0.05969(2) d, assuming that the superhump phase and period did not change during a normal outburst.

\subsection{Relation between $r_{p o s t}$ and $q$}

The dynamical precession rate in the disk can be expressed using $q$ and the dimensionless radius, $r$, measured in units of the binary separation a by

$$
\omega_{\text {dyn }} / \omega_{\text {orb }}=Q(q) / R(r)
$$

where $\omega_{o r b}$ is the angular orbital frequency. For the dependencies on $q$ and $r$, see [22]. This $\omega_{d y n} / \omega_{o r b}$ is equal to the fractional superhump excess in frequency: $\varepsilon \equiv 1-P_{o r b} / P_{S H}$, where $P_{\text {orb }}$ and $P_{S H}$ are the orbital period and superhump period, respectively. If we know $P_{o r b}$, we can directly determine $q$ from the observed ieta of stage A superhumps under the assumption that the period of stage A superhumps reflects the purely dynamical precession rate at the radius of the $3: 1$ resonance [13].

The orbital period of RZ LMi, however, is not known, thus we cannot directly determin $q$ with the above method. Instead, we can use the period of post superoutburst superhumps to constrain $q$ and the disk radius as introduced in [23]:

$$
\begin{gathered}
\varepsilon^{*}(\text { stage } A)=Q(q) R\left(r_{3: 1}\right), \\
\varepsilon^{*}(\text { post })=Q(q) R\left(r_{\text {post }}\right),
\end{gathered}
$$

where $r_{3: 1}$ is the radius of the $3: 1$ resonance;

$$
r_{3: 1}=3^{(-2 / 3)}(1+q)^{-1 / 3}
$$

$\varepsilon^{*}($ post $)$ and $r_{\text {post }}$ are the fractional superhump excess and disk radius immediately after the outburst, respectively.

By solving equations (4) and (5) simultaneously using the periods of stage A superhumps and post-superoutburst superhumps, we can obtain the relation between $r_{p o s t}$ and $q$ shown in figure 4 . 
The values of $r$ - post in SU UMa-type DNe are estimated to be 0.30-0.38 using the same method [13]. The smaller values are those for WZ Sge-type DNe with small mass transfer rates, which is unlikely to be the case for RZ LMi. [9] assumed $r_{\text {post }}=0.42$ for this particular object, which requires $q$ to be as large as 0.10(2). Though [9] and [10] predict a very small $q$ and a large $r_{\text {post }}$ for $\mathrm{RZ} \mathrm{LMi}$, our result indicates the predictions are not true at the same time.

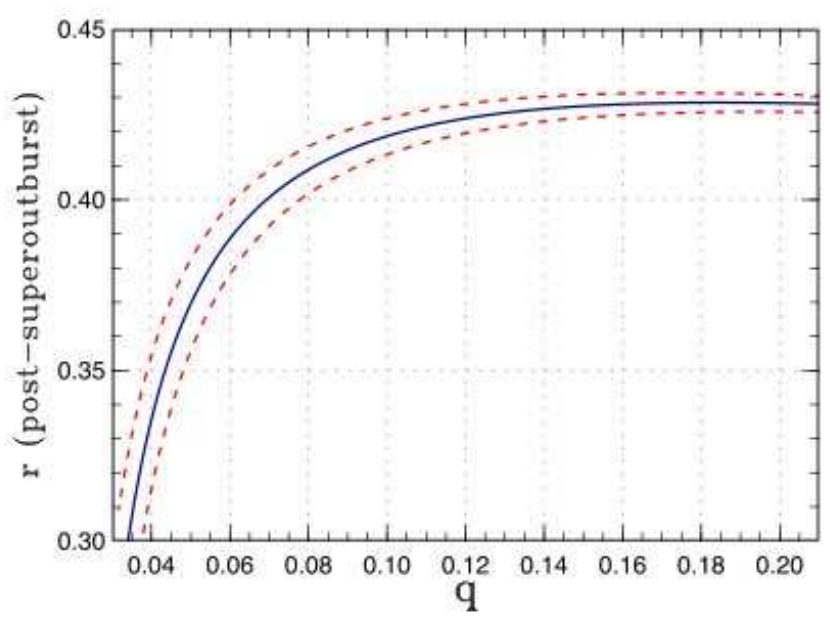

Figure 4: Relation between $q$ and $r_{\text {post }}$ derived from the periods of stage A superhumps and postsuperoutburst superhumps. (Fig. 10 in [7])

\subsection{Negative superhumps}

We analysed the light curves using the least absolute shrinkage and selection operator (Lasso) method ([24]; [25]). Two-dimensional Lasso power spectra introduced in the analysis of the Kepler data as [26] and [12] have been proven to be very effective in detecting signals in non-uniformly sampled ground-based data (see e.g., [28]).

Figure 5 shows the result for RZ LMi in 2016. In addition to the strong persistent signal of superhumps around 16.8 cycles per day $\left(\mathrm{cd}^{-1}\right)$, there is a weaker signal around $17.50-17.55 \mathrm{~cd}^{-1}$ between BJD2457510 and 2457530, that is a later phase of the long-lasting superoutburst (2016$\mathrm{SC} 2$ ). We consider that the signal is a possible negative superhumps. A PDM analysis of the data between BJD 2457510 and 2457530 yielded a period of 0.05710(1) $\mathrm{d}$. The superhump period in this interval was $0.059555(4) \mathrm{d}$. Negative superhumps are often observed in permanent superhumpers. It may be possible that negative superhumps in RZ LMi were excited during the phase in which the condition of the object was almost the same as that in permanent superhumpers (see subsection $3.1)$.

\subsection{Orbital period and mass ratio}

There is an empirical relation between the absolute superhumps excesses $\left(\varepsilon \equiv P_{S H} / P_{\text {orb }}-1\right)$ of negative and positive superhumps in NL objects. The relation is $\varepsilon_{+} \sim 2\left|\varepsilon_{-}\right|$, where $\varepsilon_{+}$for positive superhumps and $\varepsilon_{-}$for negative superhumps. If this is alsk the case for RZ LMi, we obtain the orbital period of $0.05792 \mathrm{~d}$. This period is labeled as $P_{\text {orb }}$ in figure 5 . 


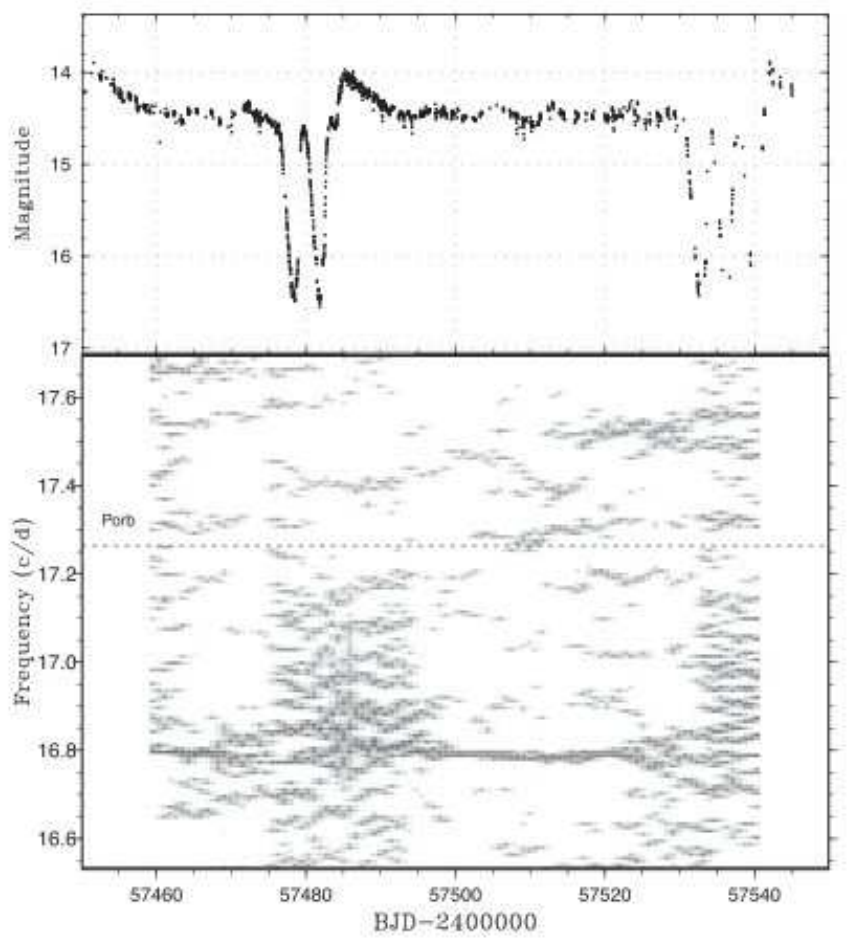

Figure 5: Two-dimensional Lasso period analysis of the 2016 light curve. Upper: Light curve. Lower: Lassoperiod analysis.the strong signal around $16.8 \mathrm{c} / \mathrm{d}$ is superhumps. A weaker signal around 17.0-17.5 c/d between BJD 2457510 and 2457530 is possible negative superhumps. (Fig. 11 in [7])

If this is indeed the orbital period, the period of stage A superhumps of 0.602(1) d gives $\varepsilon=0.038(2)$, which is equivalent to $q=0.105(5)$ [see table 1 in [13]]. The $q$ is consistent with the relation between $q$ and $r_{\text {post }}$ derived from the periods of stage A superhumps and negative superhumps in subsection 4.2 (figure 4) assuming the large $r_{\text {post }}$. If this is correct, the disk radius at the end of a superoutburst is large, as required by [9], but the large disk cannot be explained by an exceptionally small $q$.

A supporting evidence for a large $q$ is the very short duration (less than $1 \mathrm{~d}$ ) of stage A superhumps in RZ LMi. The duration is considered to reflect the growth time of the 3:1 resonance, and expected to be proportional to $1 / q^{2}$ [29], which has been confirmed in WZ Sge-type DNe [30]. The rapid growth of superhumps in RZ LMi indicates that the $q$ of this object cannot be as small as those in WZ Sge-type DNe.

\subsection{Evolutionary status}

If the $q$ derived in subsection 3.6 is correct, RZ LMi cannot be an object close to the period minimum or a period bouncer. The $q$ we suggested is similar to or even larger than those of ordinary SU UMa stars with similar $P_{\text {orb }}$ (Figure 6).

There is at least one other object, GALEX J194419.33+491257.0, with extremely high $q=$ 0.141 (2) for a very short $P_{\text {orb }}$ of 0.0528164(4) d and very frequent outbursts [27]. These properties are somehow similar to those of RZ LMi and [27] suggested the possibility that this object may be 
a CV with a stripped core-evolved secondary evolving toward an AM CVn-type CV. This condition might be a possible explanation of RZ LMi with an exceptionally high $\dot{M}$ for its $P_{\text {orb }}$.

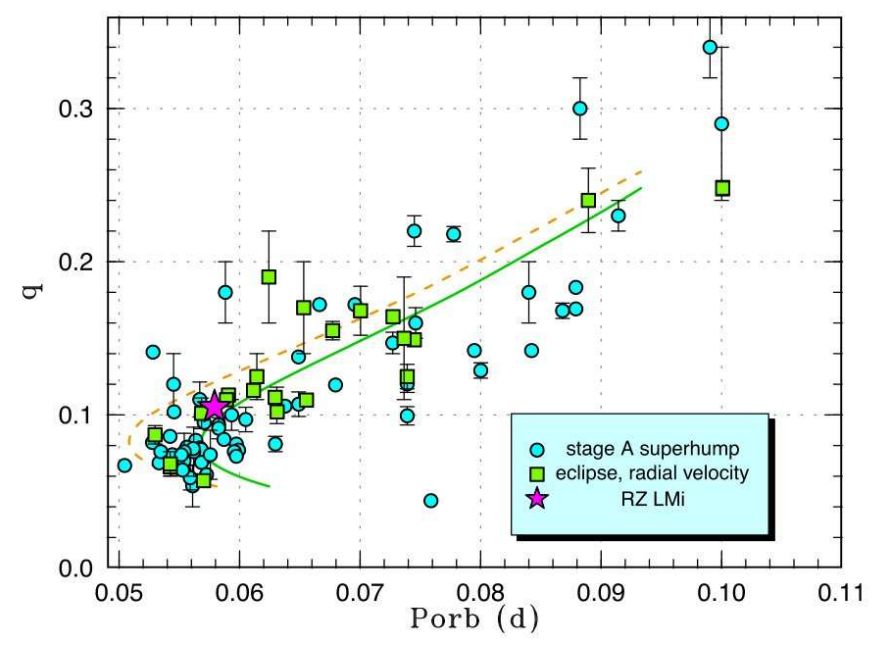

Figure 6: Location of RZ LMi on the diagram of mass ratio versus orbital period. (Fig. 12 in [7])

\subsection{Secular variation of supercycle}

There is an idea that ER UMa stars are transitional objects during the cooling phase of posteruption classical novae ([6], [9], [19]). A transition from the NL (permanent superhumper) state to the ERUMa-type state discovered in BK Lyn was thought to support the idea, combined with the potential identification with an ancient classical nova in 101 [19]. Following this interpretaion, [31] found a secular increase of the supercycle in most ER UMa-type objects including RZ LMi.

They, however, disregarded the possibility that the supercycle can also increase if $\dot{M}$ increases toward $\dot{M}_{\text {crit }}$ (see subsection 3.1), which is apparently the case for RZ LMi. We studied the secular variation of supercycles in RZ LMi, and found that the supercycle was not stable or secularly increasing, but variable. As discussed in subsection 3.1, the supercycle of RZ LMi strongly increased in 2016, and the increase was due to the increase of $\dot{M}$. It is likely that RZ LMi changed $\dot{M}$ by a factor of $\sim 2$ in the last two decades. The changing supercycle suggests fluctuating $\dot{M}$ within time scales of a few years.

In RZ LMi, the mass transfer rate is not secularly decreasing as in the scenario given by [19]. BK Lyn also returned to ints original NL state in late 2013 from its temporal ER UMa-type state [20]. The hypothetical cooling sequence from NL objects to SU UMa-type DNe via ER UMa-type DNe after nova eruptions is not very consistent with observational staticsics. The Mdot variations look more irregular with time-scales of several years. The high activity of RZ LMi may be a result of a rare evolutionary condition with a relatively massive secondary.

\section{References}

[1] J. Smak, Accretion in cataclysmic binaries. IV - Accretion disks in dwarf novae, Acta Astron 34 (1995) 161. 
[2] B. Warner, Cataclysmic Variable Stars, PublisherCambridge, 1995.

[3] V. A. Lipovetskii and J. A. StepanyanAstrofizika 17 (1981) 573.

[4] J. W. Robertson, R. K. Honeycutt and G. W. Turner, RZ Leonis Minoris, PG 0943+521, and V1159 Orionis: Three cataclysmic variables with similar and unusual outburst behavior, PASP 107 (1995) 443-449.

[5] D. Nogami, T. Kato, S. Masuda, R. Hirata, K. Matsumoto, K. Tanabe et al., Photometric observations of an extreme ER UMa star, RZ Leonis Minoris, PASJ 47 (1995) 897.

[6] T. Kato and C. Kunjaya, Discovery of a peculiar SU UMa-type dwarf nova ER Ursae Majoris, PASJ 47 (1995) 163.

[7] T. Kato, R. Ishioka, K. Isogai, M. Kimura, A. Imada, I. Miller et al., Rz leonis minoris bridging between er ursae majoris-type dwarf nova and nova-like system, PASJ 68 (2016) 107.

[8] T. Kato, D. Nogami, H. Baba, S. Masuda, K. Matsumoto and C. Kunjaya, Observation of ER UMa stars, in Disk Instabilities in Close Binary Systems (S. Mineshige and J. C. Wheeler, eds.), p. 45, PublisherUAP, 1999.

[9] Y. Osaki, Why does rz leonis minoris, and unusual su uma star, have such a short supercycle?, PASJ 47 (1995) L25.

[10] C. Hellier, On echo outbursts and er uma supercycles in su uma-type cataclysmic variables, PASP 113 (2001) 469.

[11] A. Olech, M. Wisniewski, K. Zloczewski, L. M. Cook, K. Mularczyk and P. Kedzierski, Curious Variables Experiment (CURVE). RZ LMi - the most active SU UMa star, Acta Astron 58 (2008) 131.

[12] Y. Osaki and T. Kato, Study of superoutbursts and superhumps in SU UMa stars by the Kepler light curves of V344 Lyrae and V1504 Cygni, PASJ 65 (2013) 95.

[13] T. Kato and Y. Osaki, New method of estimating binary's mass ratios by using superhumps, PASJ 65 (2013) 115.

[14] O. D. Pikalova and S. Y. Shugarov, The photometric investigation of RZ LMi, in Cataclysmic Variables (A. Bianchini, M. della Valle and M. Orio, eds.), p. 173, PublisherKluwer, 1995.

[15] T. Kato, A. Imada, M. Uemura, D. Nogami, H. Maehara, R. Ishioka et al., Survey of Period Variations of Superhumps in SU UMa-Type Dwarf Novae, PASJ 61 (2009) S395.

[16] T. Kato, P. A. Dubovsky and I. Kudzej, Survey of period variations of superhumps in su uma-type dwarf novae. vi. the sixth year (2013-2014), PASJ 66 (2014) 90.

[17] J. W. Robertson, R. K. Honeycutt, G. W. Turner, The Unsusual Long-Term Repetitive Light Curve of $R Z$ Leonis Minoris, in proceedings of n ASP Conf. Ser. 56, Interacting binary stars, (A. W. Shafter, ed), p. 298, PublisherASP, 1994.

[18] Y. Osaki, A model for a peculiar SU Ursae Majoris-type dwarf nova ER Ursae Majoris, PASJ 47 (1995) L11-L14.

[19] J. Patterson, H. Uthas, J. Kemp, E. de Miguel, T. Krajci, J. Foote et al., BK Lyncis: the oldest old nova and a Bellwether for cataclysmic variable evolution, MNRAS 434 (2013) 1902.

[20] T. Kato, F-J. Hambsch, H. Maehara, G. Masi, I. Miller, R. Noguchi et al., Survey of Period Variations of Superhumps in SU UMa-Type Dwarf Novae. IV. The Fourth Year (2011-2012), PASJ 65 (2013) 23. 
[21] R. F. Stellingwerf, Period determination using phase dispersion minimization, ApJ 224 (1978) 953-960.

[22] M. Hirose and Y. Osaki, Hydrodynamic simulations of accretion disks in cataclysmic variables superhump phenomenon in su uma stars, PASJ 42 (1990) 135.

[23] T. Kato and B. Monard, Sss j122221.7-311523: Double superoutburst in the best candidate for a period bouncer, PASJ 65 (2013) L11.

[24] R. Tibshirani, Regression shrinkage and selection via the lasso, J. R. Statist. Soc. B 58 (1996) 267.

[25] T. Kato and M. Uemura, Period analysis using the Least Absolute Shrinkage and Selection Operator (Lasso), PASJ 64 (2012) 122.

[26] T. Kato and H. Maehara, Analysis of Kepler light curve of the novalike cataclysmic variable KIC 8751494, PASJ 65 (2013) 76.

[27] T. Kato and Y. Osaki, GALEX J194419.33+491257.0: An unusually active SU UMa-type dwarf nova with a very short orbital period in the Kepler data, PASJ 66 (2014) 5.

[28] T. Ohshima, T. Kato, E. Pavlenko, H. Akazawa, K. Imamura, K. Tanabe et al., Study of negative and positive superhumps in ER Ursae Majoris, PASJ 66 (2014) 67.

[29] S. H. Lubow, A model for tidally driven eccentric instabilities in fluid disks, ApJ 381 (1991) 259.

[30] T. Kato, WZ Sge-type dwarf novae, PASJ 67 (2015) 105.

[31] M. Otulakowska-Hypka, A. Olech, On supercycle lengths of active SU UMa stars, MNRAS 433 (2012) 1338.

\section{DISCUSSION}

VOJTECH SIMON: Was always the time segment between the superoutbursts filled by dense series of normal outbursts or were any segments longer than the short ones filled mainly by the quiessent state (with only a few normal outbursts)?

RYOKO ISHIOKA: As for RZ LMi, the ourbust parttern is basically very regular. One superoutbursts followed by two normal outbursts with supercycle of about $20 \mathrm{~d}$. When a supercycle becomes longer, it seems to be due to a longer superoutburst, so the system is not quiet. The extreme case was what is observed in 2016. For some other objetcts, it is suggested or confirmed that the system is quiet ( with less normal outbursts) when there exist negative superhumps. 